\title{
HIGH FREQUENCY INDUCED INSTABILITY IN NYSTRÖM METHODS FOR THE VAN DER POL EQUATION*
}

\author{
S. W. SCHOOMBIE ${ }^{\dagger}$ AND E. MARÉ $\ddagger$
}

\begin{abstract}
In this paper several Nyström methods for the van der Pol equation are considered. In an earlier study by Cai, Aoyagi, and Abe it was shown that the second order Nyström, or leapfrog, method fails to approximate the limit cycle of the van der Pol equation, exhibiting a periodic modulation of the amplitude and sporadic high frequency noise instead. Cai et al. did a linear analysis and concluded that the spurious behavior was due to the interaction of the main part of the solution with a high frequency computational mode. In this paper we also apply a third and fourth order Nyström method to the van der Pol equation. Numerical experiments show that in these cases the high frequency mode causes blowup after some time. The onset of the instability can be delayed by decreasing the time step. We also improve on their analysis of the second order scheme by doing a nonlinear analysis, to wit a discrete multiple scales analysis. By this means we are able to explain the spurious behavior of this system completely.
\end{abstract}

Key words. van der Pol equation, spurious solutions, Nyström, leapfrog, multiple scales, discrete approximation, difference equations

AMS subject classifications. 74H15, 74H55, 93C $70,65 \mathrm{~L} 07,65 \mathrm{P} 40$

DOI. $10.1137 / 050644148$

1. Introduction. The van der Pol equation

$$
\frac{d^{2} x}{d t^{2}}-\epsilon\left(1-x^{2}\right) \frac{d x}{d t}+\omega^{2} x=0
$$

with

$$
0<\epsilon \ll 1
$$

was first formulated by van der Pol [12] in 1922 in the context of electronic circuits in vacuum tubes.

This equation is well known to have an unstable equilibrium at the origin of its phase plane, surrounded by a stable limit cycle. It is a standard classroom exercise to find an approximate analytic expression for the solution of the equation, using a multiple scales method [3,6], namely,

$$
x=\frac{2 \cos (\omega t+\phi)}{\sqrt{\left.1+\left(4 / a_{0}^{2}-1\right) e^{-\epsilon t}\right)}}+O(\epsilon),
$$

where $a_{0}$ is the initial amplitude.

Thus the limit cycle has a period which is very near to $2 \pi / \omega$ and an amplitude which is very near to 2 . To find the limit cycle more precisely, the van der Pol equation needs to be solved numerically. Various approaches have been used for this purpose.

*Received by the editors November 3, 2005; accepted for publication (in revised form) November 16, 2007; published electronically March 7, 2008

http://www.siam.org/journals/sinum/46-3/64414.html

${ }^{\dagger}$ Department of Mathematics and Applied Mathematics, University of the Free State, P.O. Box 339, Bloemfontein 9300, Republic of South Africa (schoomsw.sci@ufs.ac.za).

${ }_{\ddagger}^{\ddagger}$ Department Mathematics and Applied Mathematics, University of Pretoria, Pretoria 0002, Republic of South Africa (eben.mare@up.ac.za). This work was carried out while this author was affiliated with the University of the Free State. 
These include extrapolation-based methods with step size control [2], Runge-Kutta Nyström methods [2], the implicit Adams method of order up to twelve, and Gear's method for stiff equations [4], among others.

That care must be taken when designing a high order finite difference method for the van der Pol equation was shown by Cai, Aoyagi, and Abe [1]. They considered a second order Nyström method which showed a peculiar type of spurious behavior. Its solution periodically approaches the correct limit cycle, but then moves away from it again. They essentially used a linear analysis to explain this phenomenon, ascribing the spurious behavior to a spurious mode which interferes with the numerical solution.

We investigated this phenomenon further by also applying third and fourth order Nyström methods to the van der Pol equation. In these cases we found that the solution behaved reasonably well for some time, after which there was rapid growth of a high frequency mode, followed by blowup. By decreasing the time step the onset of this nonlinear instability may be delayed.

To analyze this type of instability, we considered only the simplest case, namely, the second order Nyström, or leapfrog, method used by Cai et al. [1]. We felt that their linear analysis provides only part of the explanation, and that a nonlinear analysis would give a more complete picture. This paper attempts to do just that. We use a discrete version of the multiple scales technique mentioned above to analyze the difference equation, and finally show that the full explanation is to be found in the phase plane.

In section 2 we will briefly review the difference scheme considered by Cai et al. [1] and demonstrate the nature of its spurious behavior, as well as show the behavior of the third and fourth order Nyström schemes.

In sections 3 and 4 we then proceed with a nonlinear analysis to explain the observed phenomena in the case of the second order scheme. The results of the nonlinear analysis is then related to some numerical results in section 5 . Section 6 contains some conclusive remarks.

2. Nyström time discretizations. Following [1], we rewrite (1.1) as a system of two first order equations, namely,

$$
\begin{aligned}
& \frac{d x}{d t}=y, \\
& \frac{d y}{d t}=\epsilon\left(1-x^{2}\right) y-\omega^{2} x .
\end{aligned}
$$

To discretize these equations, we introduce the time step $\tau$, the finite difference approximations $x^{n} \approx x(n \tau)$ and $y^{n} \approx y(n \tau)$, and the shift operators $E$ and $E^{-1}$, defined for any sequence $x^{n}$ by

$$
E x^{n}=x^{n+1} \quad \text { and } \quad E^{-1} x^{n}=x^{n-1} .
$$

Finally, we define the central divided difference operator

$$
\delta=\left(E-E^{-1}\right) /(2 \tau) .
$$

2.1. Second order Nyström discretization (leapfrog). Following Cai et al. [1], we first discretize the system (2.1), (2.2) by means of a second order Nyström method:

$$
\begin{aligned}
& \delta x^{n}=y^{n}, \\
& \delta y^{n}=\epsilon\left(1-\left(x^{n}\right)^{2}\right) y^{n}-\omega^{2} x^{n} .
\end{aligned}
$$

Copyright (C) by SIAM. Unauthorized reproduction of this article is prohibited. 


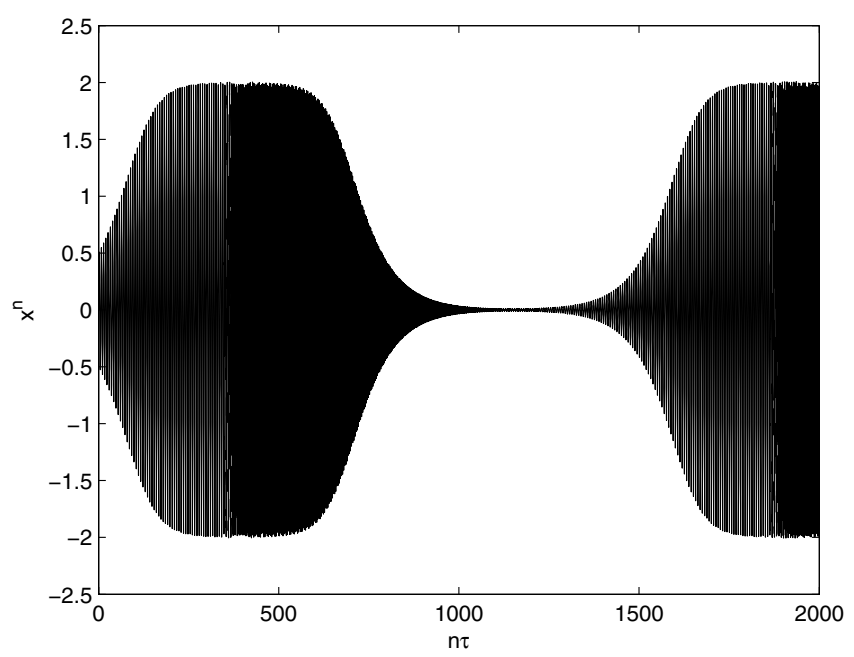

FIG. 1. Results when running the difference system (2.5), (2.6) with $\epsilon=0.025, \omega=1, \tau=0.2$, and $x^{0}=0, y^{0}=0.5$.

This discretization is often referred to as a leapfrog method.

The system of difference equations (2.5), (2.6) can easily be seen to be equivalent to a single fourth order difference equation,

$$
\begin{aligned}
& \left(E^{2}-2+E^{-2}\right) x^{n} /\left(4 \tau^{2}\right) \\
& -\epsilon\left(1-\left(x^{n}\right)^{2}\right)\left(E-E^{-1}\right) x^{n} /(2 \tau)+\omega^{2} x^{n}=0 .
\end{aligned}
$$

Using the following notation for the forward and backward divided difference operators, respectively,

$$
\Delta=(E-1) / \tau, \quad \nabla=\left(1-E^{-1}\right) / \tau
$$

we can rewrite $(2.7)$ in the more concise form

$$
\left[\Delta \nabla+\frac{\tau^{2}}{4} \Delta^{2} \nabla^{2}\right] x^{n}-\epsilon\left(1-\left(x^{n}\right)^{2}\right) \delta x^{n}+\omega^{2} x^{n}=0 .
$$

To compute a numerical solution from (2.5) and (2.6), we need to specify initial values $x^{0}$ and $y^{0}$. Values for $x^{1}$ and $y^{1}$ are also required, and these are calculated by means of a second order Runge-Kutta starter with time increment $\tau$. Figure 1 shows the result of such a computation with $\epsilon=0.025, \omega=1, \tau=0.2$, and $x^{0}=0, y^{0}=0.5$. Initially everything seems to go well, and the solution approaches the limit cycle with its amplitude at around 2 and remains with it for a while. However, after some time it leaves the limit cycle and eventually returns to it again. What seems to be a periodic amplitude modulation is imposed, which is quite spurious.

Cai et al. [1] showed that this spurious behavior is due to the presence of a high frequency computational mode. They performed a linear stability analysis and showed that the computational mode starts growing when the amplitude of the numerical solution reaches a value of about $\sqrt{0.8}$.

The onset of the growth of the high frequency mode can be delayed by decreasing the time step, as shown in Figure 2, where the time step was decreased to $\tau=0.1$.

Copyright (C) by SIAM. Unauthorized reproduction of this article is prohibited. 


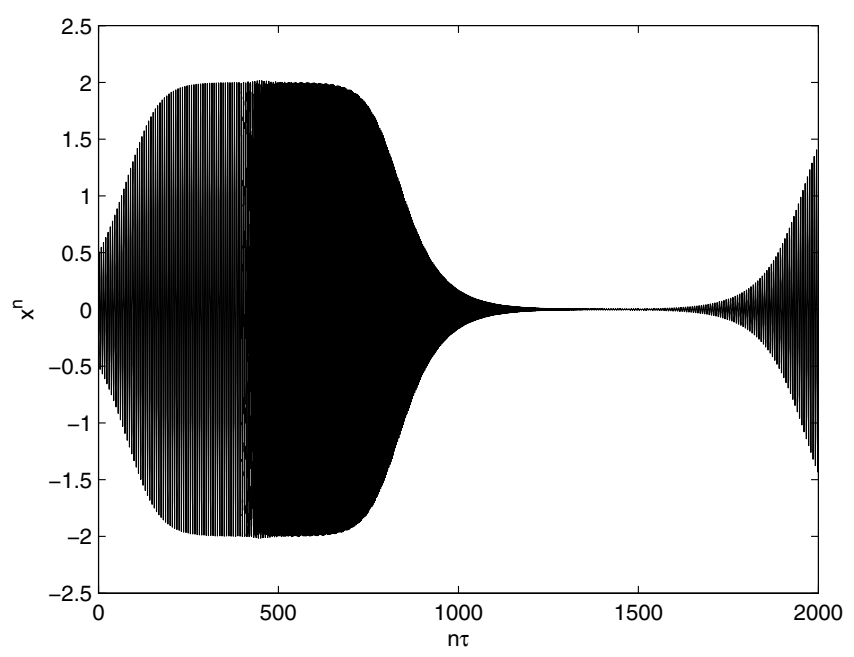

FIG. 2. Results when running the difference system (2.5), (2.6) with $\epsilon=0.025, \omega=1, \tau=0.1$, and $x^{0}=0, y^{0}=0.5$.

2.2. Third order Nyström time discretization. The order of convergence of the numerical method can be increased by using a higher order Nyström discretization. For a third order scheme the discretization of the system becomes

$$
\begin{aligned}
\delta x^{n} & =\frac{1}{6}\left[7 y^{n}-2 y^{n-1}+y^{n-2}\right] \\
\delta y^{n} & =\frac{1}{6}\left[7 f\left(x^{n}, y^{n}\right)-2 f\left(x^{n-1}, y^{n-1}\right)+f\left(x^{n-2}, y^{n-2}\right)\right],
\end{aligned}
$$

where

$$
f(x, y)=\epsilon\left(1-x^{2}\right) y-\omega^{2} x .
$$

This time we need to supply two extra initial values, namely, for both $n=1$ and $n=2$. Because of the increased order of this method we used a fourth order Runge-Kutta method to calculate these initial values.

Using the same parameters as in Figure 1, we find that the numerical solution does not stay finite though spuriously modulated as with the leapfrog discretization, but that it suddenly blows up. The reason for this instability is the same, however: a sudden growth of a high frequency mode. The result is shown in Figure 3.

Since this is a convergent method, like the leapfrog method above, the onset of the instability can again be delayed by using a smaller time step. In order to reach the limit cycle before the high frequency mode grows significantly, we found that a time step of at most $\tau=0.05$ has to be used.

Thus, although a higher order method is used, a much smaller time step is required in order to find the limit cycle numerically. The high frequency induced (HFI) instability observed here enforces an upper limit to the time step.

Copyright (C) by SIAM. Unauthorized reproduction of this article is prohibited. 


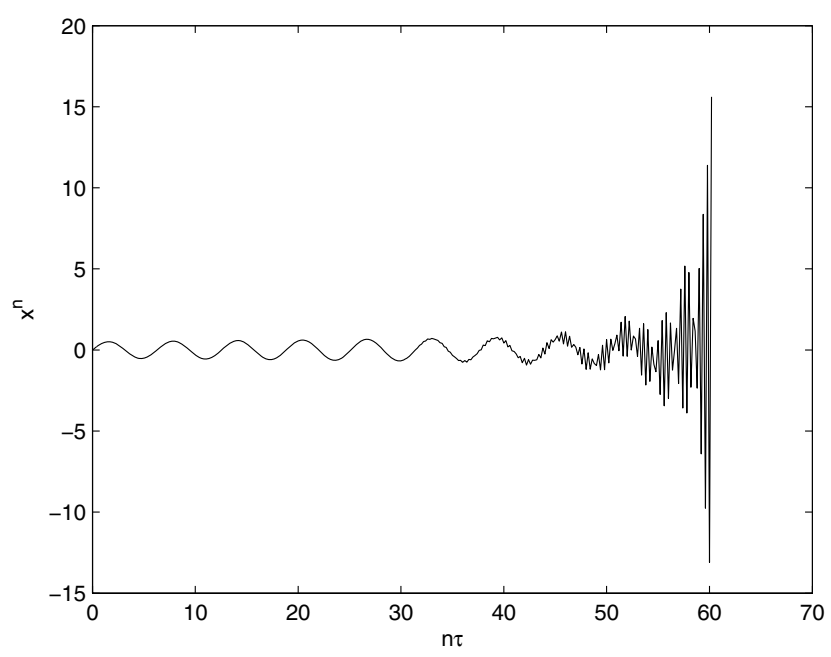

FIG. 3. Results when running the difference system (2.10), (2.11) with $\epsilon=0.025, \omega=1, \tau=0.2$, and $x^{0}=0, y^{0}=0.5$.

2.3. Fourth order Nyström time discretization. Applying the fourth order Nyström discretization to the system (2.1), (2.2), we get

$$
\begin{aligned}
\delta x^{n} & =\frac{1}{6}\left[8 y^{n}-5 y^{n-1}+4 y^{n-2}-y^{n-3}\right] \\
\delta y^{n} & =\frac{1}{6}\left[8 f\left(x^{n}, y^{n}\right)-5 f\left(x^{n-1}, y^{n-1}\right)+4 f\left(x^{n-2}, y^{n-2}\right)-f\left(x^{n-3}, y^{n-3}\right)\right]
\end{aligned}
$$

with $f$ as in (2.12).

The three extra initial values are again computed with a fourth order RungeKutta method. The results are similar to those of the third order method, except that the high frequency mode now appears much sooner, again leading to blowup. Figure 4 shows what happens when $\tau=0.05$, and it turns out that the limit cycle can be reached fairly accurately only for time steps lower than about $\tau=0.01$. Thus the upper bound for the time step is even lower than before.

Having observed the havoc caused by the high frequency spurious mode, the next thing to be attempted is an analysis of this phenomenon. This is done in the next section for the leapfrog case.

3. A discrete multiple scales analysis. Since the behavior of the leapfrog time discretization is the most complicated (the others simply blow up), and since this is also the simplest difference scheme, we shall attempt an analysis of this case only.

Since the spurious behavior of (2.9) consists of an amplitude modulation, a multiple scales analysis would seem to be an appropriate tool to investigate it.

To perform a multiple scales analysis of (2.9) we shall work in direct analogy with the well-known continuous multiple scales analysis performed for the van der Pol equation, e.g., [6]. We shall use two discrete time scales, namely,

$$
T_{p}=\epsilon^{p} n \tau, \quad p=0,1
$$

Copyright (C) by SIAM. Unauthorized reproduction of this article is prohibited. 


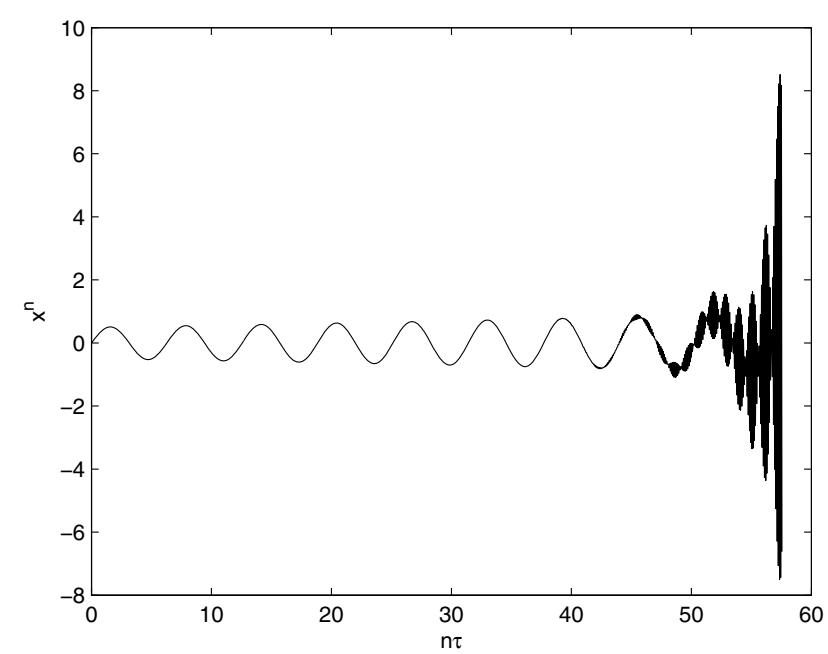

FIG. 4. Results when running the difference system (2.13), (2.14) with $\epsilon=0.025, \omega=1$, $\tau=0.05$, and $x^{0}=0, y^{0}=0.5$.

and consider the expansion [9]

$$
x^{n}=x_{0}\left(T_{0}, T_{1}\right)+\epsilon x_{1}\left(T_{0}, T_{1}\right)+O\left(\epsilon^{2}\right) .
$$

We shall also use partial shift operators $E_{T_{p}}$ which we define as

$$
E_{T_{p}} f\left(\ldots, T_{p}, \ldots\right):=f\left(\ldots, T_{p}+\epsilon^{p} \tau, \ldots\right)
$$

in analogy with the temporal shift operator in (2.3). Using the partial shift operator (3.3) above we also define partial divided difference operators for $T_{p}$, namely,

$$
\Delta_{T_{p}}:=\left(E_{T_{p}}-1\right) /\left(\epsilon^{p} \tau\right), \quad \nabla_{T_{p}}:=\left(1-E_{T_{p}}^{-1}\right) /\left(\epsilon^{p} \tau\right)
$$

The analogue of the central difference operator $\delta$, defined in (2.4), follows similarly:

$$
\delta_{T_{p}} \equiv\left(\Delta_{T_{p}}+\nabla_{T_{p}}\right) / 2
$$

Following Schoombie [9, 10] we note the following discrete multiple scales expansion of the differential approximations $\Delta$ and $\nabla$ given in (2.8), namely,

$$
\Delta=\Delta_{T_{0}}+\epsilon \Delta_{T_{1}} E_{T_{0}}, \quad \nabla=\nabla_{T_{0}}+\epsilon \nabla_{T_{1}} E_{T_{0}}^{-1}
$$

By making use of the definition of $\delta$ in (2.4) and substitution of (3.6) therein, we also obtain that

$$
\delta=\delta_{T_{0}}+\epsilon / 2\left(\Delta_{T_{1}} E_{T_{0}}+\nabla_{T_{1}} E_{T_{0}}^{-1}\right) .
$$

The equations above are essentially the discrete analogues of the chain rule for differentiation as used in a normal continuous multiple scales analysis. 
We now proceed with our discrete multiple scales analysis. We substitute (3.6) into (2.9). Note the expansion of the first operator in (2.9):

$$
\begin{aligned}
& \Delta \nabla+\left(\tau^{2} / 4\right) \Delta^{2} \nabla^{2} \\
& =\Delta_{T_{0}} \nabla_{T_{0}}+\left(\tau^{2} / 4\right) \Delta_{T_{0}}^{2} \nabla_{T_{0}}^{2} \\
& +\epsilon\left(\Delta_{T_{0}} \Delta_{T_{1}}+\nabla_{T_{0}} \nabla_{T_{1}}+\left(\tau^{2} / 2\right) \Delta_{T_{0}}^{2} \nabla_{T_{0}} \Delta_{T_{1}}+\left(\tau^{2} / 2\right) \nabla_{T_{0}}^{2} \Delta_{T_{0}} \nabla_{T_{1}}\right) .
\end{aligned}
$$

We proceed by collecting terms with equal powers of $\epsilon$. In this way we generate a set of equations given by

$$
L x_{0}=0,
$$

relevant to $O(1)$, and for $O(\epsilon)$,

$$
L x_{1}\left(T_{0}, T_{1}\right)=R\left(T_{0}, T_{1}\right),
$$

where the difference operator $L$ is defined by

$$
L u\left(T_{0}, T_{1}\right)=\left(\Delta_{T_{0}} \nabla_{T_{0}}+\left(\tau^{2} / 4\right) \Delta_{T_{0}}^{2} \nabla_{T_{0}}^{2}\right) u\left(T_{0}, T_{1}\right)+\omega^{2} u\left(T_{0}, T_{1}\right)
$$

for any discrete variable $u\left(T_{0}, T_{1}\right)$, and where

$$
R\left(T_{0}, T_{1}\right)=-\left(\Delta_{T_{0}} \Delta_{T_{1}}+\nabla_{T_{0}} \nabla_{T_{1}}+\left(\tau^{2} / 2\right) \Delta_{T_{0}}^{2} \nabla_{T_{0}} \Delta_{T_{1}}+\left(\tau^{2} / 2\right) \nabla_{T_{0}}^{2} \Delta_{T_{0}} \nabla_{T_{1}}\right) x_{0}
$$

$$
+\beta\left(1-x_{0}^{2}\right) \delta_{T_{0}} x_{0} .
$$

It is easy to see that any real-valued solution of (3.9) must be of the form

$$
x_{0}\left(T_{0}, T_{1}\right)=a\left(T_{1}\right) e^{i \Omega n \tau / 2}+(-1)^{n} b\left(T_{1}\right) e^{i \Omega n \tau / 2}+\text { c.c. },
$$

where c.c. denotes the complex conjugate, and where

$$
\cos (\Omega \tau)=1-2 \omega^{2} \tau^{2} .
$$

The second term in (3.13) represents the computational wave considered by Cai et al. [1], the presence of which causes spurious behavior.

If we substitute this expression for $x_{0}$ into the right-hand side of (3.10), we eventually find that (neglecting $O\left(\epsilon^{2}\right)$ terms)

$$
\begin{aligned}
\frac{\tau}{i} R\left(T_{0}, T_{1}\right)= & \left\{-\sin (\Omega \tau) \delta_{T_{1}} a+\sin (\Omega \tau / 2)\left(a-a|a|^{2}+3 b^{2} \bar{a}-2 a|b|^{2}\right)\right\} e^{i \Omega n \tau / 2} \\
& +\left\{-\sin (\Omega \tau) \delta_{T_{1}} b+\sin (\Omega \tau / 2)\left(-b+b|b|^{2}-3 a^{2} \bar{b}+2 b|a|^{2}\right)\right\}(-1)^{n} e^{i \Omega n \tau / 2} \\
& +\sin (\Omega \tau / 2)\left(-a^{3}+a b^{2}\right) e^{3 i \Omega n \tau / 2} \\
& +\sin (\Omega \tau / 2)\left(b^{3}-a^{2} b\right)(-1)^{n} e^{3 i \Omega n \tau / 2}+\text { c.c. },
\end{aligned}
$$

where the bar indicates the complex conjugate.

To ensure a bounded solution for $x_{1}$, we need to remove secular terms, which is done by requiring that the coefficients of both $e^{i \Omega n \tau / 2}$ and $(-1)^{n} e^{i \Omega n \tau / 2}$ be equated to zero. This leads to the two ordinary differential equations

$$
\begin{aligned}
& a^{\prime}=r\left(a-a|a|^{2}+3 b^{2} \bar{a}-2 a|b|^{2}\right), \\
& b^{\prime}=-r\left(b-b|b|^{2}+3 a^{2} \bar{b}-2 b|a|^{2}\right),
\end{aligned}
$$

Copyright (C) by SIAM. Unauthorized reproduction of this article is prohibited. 
where

$$
r=\frac{1}{2 \cos (\Omega \tau / 2)}
$$

and we have noted from the definition of $\delta_{T_{1}}$ that we can write

$$
\delta_{T_{1}}=\left(E_{T_{1}}-E_{T_{1}}^{-1}\right) /(2 \epsilon \tau)=\partial_{T_{1}}+O\left(\epsilon^{2}\right) .
$$

Next we put

$$
a=\frac{1}{2} \alpha\left(T_{1}\right) e^{i \theta\left(T_{1}\right)}, \quad b=\frac{1}{2} \beta\left(T_{1}\right) e^{i \phi\left(T_{1}\right)},
$$

where $\alpha$ and $\beta$ are real and nonnegative, and $\theta$ and $\phi$ are real. This enables us to write $x_{0}$ in the real form

$$
x_{0}\left(T_{0}, T_{1}\right)=\alpha \cos (\Omega n \tau / 2+\theta)+(-1)^{n} \beta \cos (\Omega n \tau / 2+\phi) .
$$

When we put (3.20) into (3.16) and (3.17), and separate real and imaginary parts, we finally obtain the following coupled system of nonlinear ordinary differential equations:

$$
\begin{aligned}
\alpha^{\prime} & =r \alpha\left(1-\alpha^{2} / 4-\beta^{2} / 2\right)+\frac{3}{4} r \alpha \beta^{2} \cos (2 \phi-2 \theta), \\
\beta^{\prime} & =-r \beta\left(1-\beta^{2} / 4-\alpha^{2} / 2\right)-\frac{3}{4} r \beta \alpha^{2} \cos (2 \phi-2 \theta), \\
\theta^{\prime} & =\frac{3}{4} r \beta^{2} \sin (2 \phi-2 \theta), \\
\phi^{\prime} & =\frac{3}{4} r \alpha^{2} \sin (2 \phi-2 \theta) .
\end{aligned}
$$

4. Amplitude dynamics. The system (3.22) through (3.25) can be reduced to three equations by introducing the variable $\gamma=\cos (2 \phi-2 \theta)$ :

$$
\begin{aligned}
\alpha^{\prime} & =r \alpha\left(1-\alpha^{2} / 4-\beta^{2} / 2\right)+\frac{3}{4} r \alpha \beta^{2} \gamma, \\
\beta^{\prime} & =-r \beta\left(1-\beta^{2} / 4-\alpha^{2} / 2\right)-\frac{3}{4} r \beta \alpha^{2} \gamma, \\
\gamma^{\prime} & =\frac{3}{2} r\left(\beta^{2}-\alpha^{2}\right)\left(1-\gamma^{2}\right) .
\end{aligned}
$$

As far as the orbits of this system are concerned, we will be interested only in the orbits within the region $\alpha \geq 0, \beta \geq 0,-1 \leq \gamma \leq 1$.

First consider the orbits for which the projections on the $\alpha \beta$-plane are along the $\alpha$-axis (i.e., $\beta=0$ ). For these the system (4.1)-(4.3) reduces to the two equations

$$
\begin{aligned}
\alpha^{\prime} & =r \alpha\left(1-\alpha^{2} / 4\right), \\
\gamma^{\prime} & =-(3 / 2) r \alpha^{2}\left(1-\gamma^{2}\right) .
\end{aligned}
$$

Equation (4.4) has the solution

$$
\alpha=\frac{2}{\sqrt{1+\left(4 / \alpha_{0}^{2}-1\right) e^{-2 r T_{1}}}}=\frac{2}{\sqrt{1+\left(4 / \alpha_{0}^{2}-1\right) e^{-2 r \epsilon n \tau}}},
$$

Copyright (C) by SIAM. Unauthorized reproduction of this article is prohibited. 
where

$$
\alpha(0)=\alpha_{0},
$$

which is very close to the amplitude in (1.3), since $2 r=1+O\left(\tau^{2}\right)$ from (3.18).

Equation (4.5) shows that if $|\gamma(0)|<1, \gamma$ will decrease monotonically and eventually approach the limiting value of $\gamma=-1$. This orbit therefore ends at the unstable equilibrium point $(2,0,-1)$.

For the rest of the orbits in the quarter cylinder, it is convenient to transform to cylindrical coordinates, i.e., the coordinates $(R, \Theta, \gamma)$, where

$$
\begin{aligned}
& \alpha=R \cos \Theta, \\
& \beta=R \sin \Theta .
\end{aligned}
$$

In terms of these coordinates, the system (4.1)-(4.3) then becomes

$$
\begin{aligned}
R^{\prime} & =r R\left(1-R^{2} / 4\right) \cos (2 \Theta), \\
\Theta^{\prime} & =-r\left[1-\frac{3}{8} R^{2}(1-\gamma)\right] \sin (2 \Theta), \\
\gamma^{\prime} & =-\frac{3}{2} r R^{2}\left(1-\gamma^{2}\right) \cos (2 \Theta) .
\end{aligned}
$$

This system can be solved analytically. First we eliminate $\Theta$ by dividing (4.12) by (4.10) to get the differential equation

$$
\frac{d \gamma}{d R}=-\frac{3}{2} \frac{R\left(1-\gamma^{2}\right)}{1-R^{2} / 4}
$$

This can easily be solved by separation of variables to give the following relationship between $\gamma$ and $R$ :

$$
\gamma=\frac{C\left(1-R^{2} / 4\right)^{6}-1}{C\left(1-R^{2} / 4\right)^{6}+1}
$$

where $C$ is a constant defined by

$$
C=\frac{\left(1+\gamma_{0}\right)}{\left(1-\gamma_{0}\right)\left(1-R_{0}^{2} / 4\right)^{6}},
$$

which is positive since $-1<\gamma_{0}=\gamma(0)<1$. We note that in the limit if $R \rightarrow 2$, then $\gamma \rightarrow-1$.

Note that (4.14) defines a family of nonintersecting surfaces, which are all tangent to the curve

$$
R=2, \quad \gamma=-1, \quad 0 \leq \Theta \leq \pi / 2 .
$$

Figure 5 shows some of these surfaces.

If we now substitute the relationship (4.14) into (4.10) and (4.11), we get the system

$$
\begin{aligned}
& R^{\prime}=r R\left(1-R^{2} / 4\right) \cos (2 \Theta) \\
& \Theta^{\prime}=-r\left[1-\frac{3 R^{2}}{4\left[C\left(1-R^{2} / 4\right)^{6}+1\right]}\right] \sin (2 \Theta)
\end{aligned}
$$

Copyright (C) by SIAM. Unauthorized reproduction of this article is prohibited. 


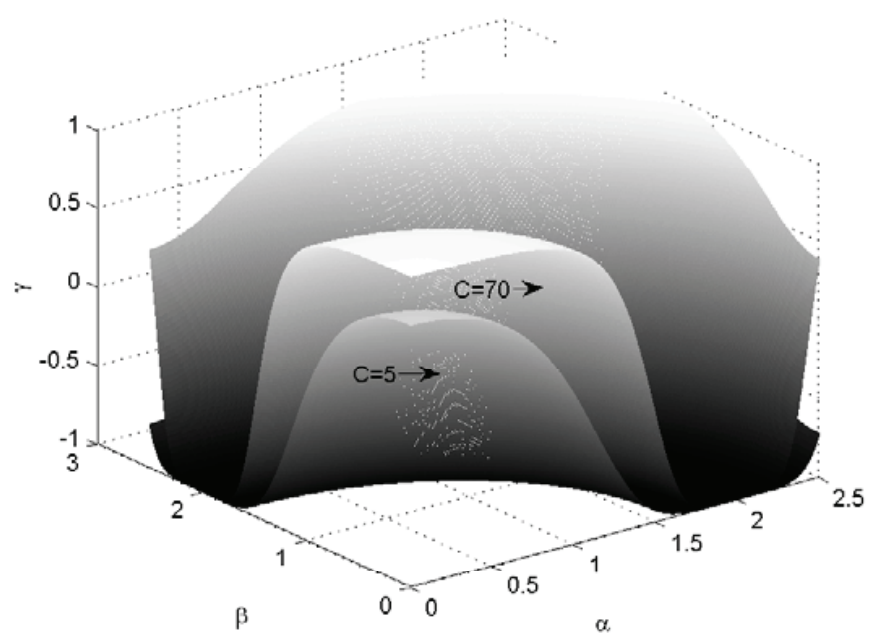

FIG. 5. Two of the surfaces defined by (4.14), namely, for $C=5$ and for $C=70$.

This system describes the projection on the $\alpha \beta$-plane of all orbits intersecting the circle arc

$$
R=R_{0}, \quad 0 \leq \Theta \leq \pi / 2, \quad \gamma=\gamma_{0},
$$

defining $C$ in (4.15).

Since we are primarily interested in the interaction of $\alpha$ and $\beta$ (and therefore that of $R$ and $\Theta$ ), this dynamical system is of particular interest to us, and it is worthwhile to examine its dynamics in some detail.

In the quadrant $0 \leq \Theta \leq \pi / 2$ the system has the equilibrium points

$$
(R, \Theta)=(0,0), \quad(2,0), \quad(2, \pi / 2),
$$

as well as the point where $\Theta=\pi / 4$ and

$$
3 R^{2}=4\left[C\left(1-R^{2} / 4\right)^{6}+1\right] .
$$

If we put $u=1-R^{2} / 4$, where $u \leq 1,(4.19)$ can be written in the simpler form

$$
C u^{6}=2-3 u \text {. }
$$

As can be seen clearly from Figure 6, the curve representing the left-hand side of (4.20) and the line representing the right-hand side have two real intersections for each value of $C$, namely, $u_{1}>0$ and $u_{2}<0$. This corresponds to two equilibrium points $\left(R_{1}, \pi / 4\right)$ and $\left(R_{2}, \pi / 4\right)$, such that $R_{1}<2$ and $R_{2}>2$. Clearly, if $C \rightarrow 0$, then $u_{1} \rightarrow 2 / 3$ and $R_{1} \rightarrow 2 / \sqrt{3}$, while $u_{2} \rightarrow-\infty$ and $R_{2} \rightarrow \infty$. Also, if $C \rightarrow \infty$, then both $u_{1}, u_{2} \rightarrow 0$ and $R_{1}, R_{2} \rightarrow 2$. Thus

$$
2 / \sqrt{3} \leq R_{1} \leq 2, \quad 2 \leq R_{2}<\infty,
$$

with the equalities achieved when $\gamma_{0}=-1$ (so that $C=0$ ) and $\gamma_{0}=1$ (so that $C=\infty)$, respectively. 


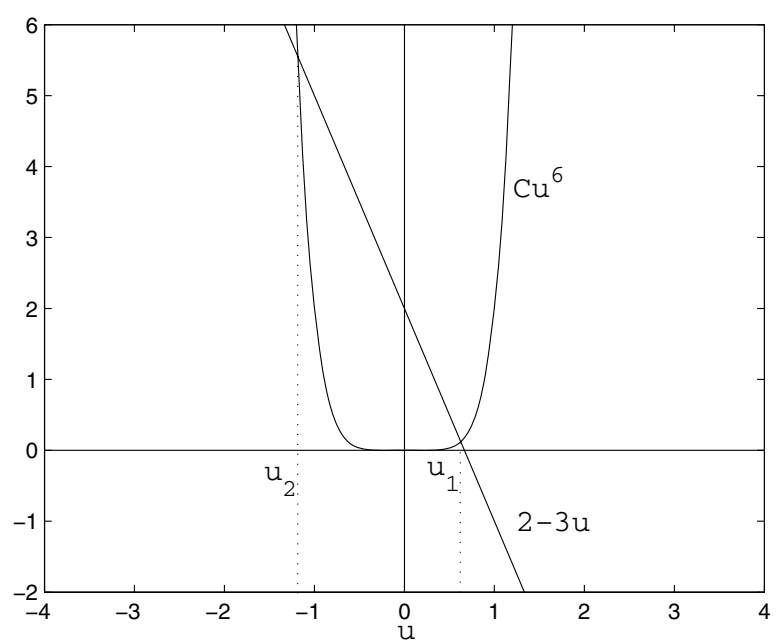

FIG. 6. The two real solutions $u_{2}<0$ and $u_{1}>0$ of (4.20), shown here for $C=2$.

The points $(R, \Theta)=(0,0),(2,0)$, and $(2, \pi / 2)$ are easily seen to be saddle points. We shall show that the remaining two equilibrium points of the projected system are both surrounded by closed orbits, and are therefore centers.

It is also easy to see that the lines $\Theta=0$ and $\Theta=\pi / 2$ are orbits, directed away from and towards the origin, respectively, as well as the circle segment $R=2$, $0 \leq \Theta \leq \pi / 2$, in a counterclockwise direction.

The rest of the orbits can be found from the differential equation

$$
\frac{d u}{d \Theta}=\frac{2 u(1-u)\left(C u^{6}+1\right) \cot (2 \Theta)}{C u^{6}+1-3(1-u)},
$$

obtained by dividing (4.16) by (4.17) and putting

$$
u=1-R^{2} / 4 \text {. }
$$

Integrating this by separation of variables yields the orbit equation

$$
\ln (\sin 2 \Theta)=\ln \frac{u}{1-u}-\frac{1}{2} \ln \left(\frac{C u^{6}}{1+C u^{6}}\right)+\text { constant, }
$$

or

$$
L \sqrt{1+C u^{6}}=u^{2}(1-u) \sin (2 \Theta)
$$

where $L$ is a constant which is nonnegative for orbits in the region under consideration. (Note that (4.24) is also true for $C=0$, i.e., $\gamma=-1$. According to (4.12) these are planar orbits.)

To investigate the nature of these orbits, we first note that (4.22) shows that for $u<1$ and $C>0$,

$$
\frac{d u}{d \Theta}=0 \text { when } \Theta=\pi / 4 .
$$

Copyright (C) by SIAM. Unauthorized reproduction of this article is prohibited. 
The extremes of $u$ are therefore on the line $\Theta=\pi / 4$ and are defined by

$$
u^{2}(1-u)=L \sqrt{1+C u^{6}}
$$

or, equivalently, are given by those zeros of the polynomial

$$
f(u)=\left(L^{2} C-1\right) u^{6}+2 u^{5}-u^{4}+L^{2}
$$

which are less than one. Now since

$$
f^{\prime}(u)=2 u^{3}\left[3\left(L^{2} C-1\right) u^{2}+5 u-2\right],
$$

the critical points of $f$ are at $u=0$ and $u=u_{ \pm}$, where

$$
u_{ \pm}=\frac{-5 \pm \sqrt{1+24 L^{2} C}}{6\left(L^{2} C-1\right)} .
$$

For all $L \geq 0, u_{+}$is always a local minimum, with

$$
0 \leq u_{+}=\frac{4}{\left(5+\sqrt{1+24 L^{2} C}\right)} \leq 2 / 3<1 .
$$

On the other hand, when $L^{2} C>1, u_{-}$is a local minimum with

$$
u_{-}=\frac{5+\sqrt{1+24 L^{2} C}}{6\left(1-L^{2} C\right)}<0,
$$

while if $L^{2} C<1, u_{-}$is a local maximum and $u_{-}>1$.

Thus the nature of $f$, and therefore of its zeros, depends very much on whether $L^{2} C>1$ or $L^{2} C<1$. If $L^{2} C<1$, i.e., for $0<L<1 / \sqrt{C}$, the graph of $f$ is as in Figure 7.

There is then always one and only one negative zero, signifying a single extreme value for $u$ and hence an open orbit in the region $R>2$. There are no positive zeros at all, unless $L \leq L_{1}$, where $L_{1}$ is that value of $L$ for which $f$ is tangent to the horizontal axis at $u_{+}$(see Figure 7(b)). For $L<L_{1}$ there are two positive zeros of $f$ less than one (Figure 7(a)), signifying two extremes in $u$ in the region $R<2$, and thus a closed orbit. When $L>L_{1}$, the function $f$ is positive for all positive $u$ (Figure $7(\mathrm{c})$ ), and hence for all $R<2$. Since this also implies that $\sin (2 \Theta)>1$, there are no orbits in this region for these values of $L$. Hence all orbits inside the quarter circle $R<2$, $0<\Theta<\pi / 2$ are closed. However, there are open orbits in the region $R>2$ for the entire range of values of $L$ between 0 and $1 / \sqrt{C}$.

When $L^{2} C>1$, i.e., $L>1 / \sqrt{C}$, the graph of $f$ is as in Figure 8 .

There are no positive zeros of $f$ less than one at all, and negative zeros only if $L<L_{2}$, where $L_{2}$ is that value of $L$ for which $f$ is tangent to the horizontal axis at $u_{-}$(Figure $\left.8(\mathrm{~b})\right)$. If $1 / \sqrt{C}<L<L_{2}$, there are two negative zeros, signifying closed orbits in the region $R>2$ (Figure $8(\mathrm{a})$ ). For $L>L_{2}$, the function $f$ is positive for all $u$ (Figure 8(c)), which means that there are no orbits at all for these values of $L$.

To find $L_{1}$ and $L_{2}$, we return to (4.26). The function $f$ will be tangent to the horizontal axis when the functions $f_{1}=u^{2}(1-u)$ and $f_{2}=L \sqrt{1+C u^{6}}$ are tangent to each other. If we suppose that the tangent point is at $u=\nu$, corresponding to $L=L^{*}$, then $\nu$ and $L^{*}$ may be found by solving the two equations

$$
\begin{aligned}
\nu^{2}(1-\nu) & =L^{*} \sqrt{1+C \nu^{6}}, \\
2 \nu-3 \nu^{2} & =\frac{3 C L^{*} \nu^{5}}{\sqrt{1+C \nu^{6}}},
\end{aligned}
$$

Copyright (C) by SIAM. Unauthorized reproduction of this article is prohibited. 

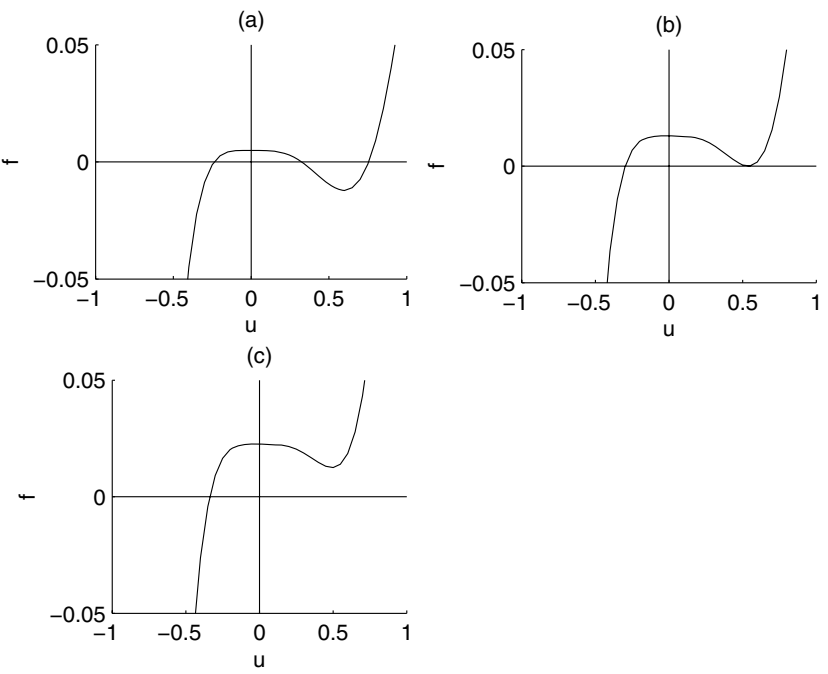

FIG. 7. The function $f$ as defined in (4.27) for $C=16$ and for (a) $L=0.07<L_{1}$, (b) $L=$ $0.1135=L_{1}$, and (c) $L=0.15>L_{1}$. In all three cases $L^{2} C<1$.

(a)

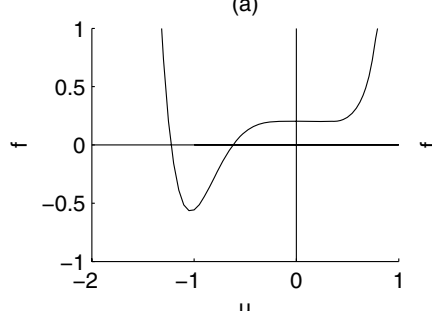

(c)

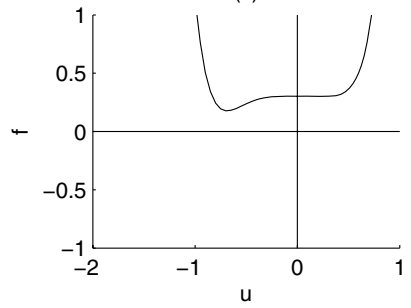

(b)

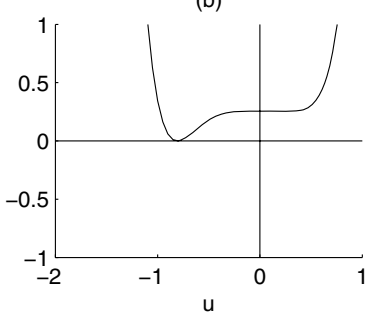

u

$=L_{2}$, and (c) $L=0.55>L_{2}$. In all three cases $L^{2} C>1$.

from which we find, by eliminating $L^{*}$, that $\nu$ satisfies

$$
2-3 \nu=C \nu^{6}
$$

which is just (4.20). Thus $\nu=u_{1}, u_{2}$ and $L^{*}=L_{1}, L_{2}$, respectively, and the tangent points are the centers $(R, \Theta)=\left(R_{i}, \pi / 4\right), i=1,2$, of the projected system. Also

$$
L_{i}=\frac{\left(u_{i}\right)^{2} \sqrt{1-u_{i}}}{\sqrt{3}} .
$$

Copyright (C) by SIAM. Unauthorized reproduction of this article is prohibited. 
(a)

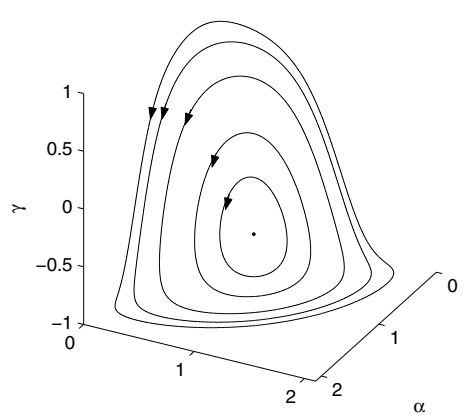

$\beta$

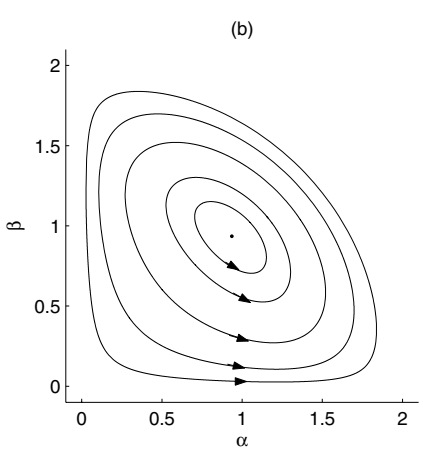

FIG. 9. Three-dimensional phase diagram (a) as well as its projection (b) on the $\alpha \beta$-plane for $C=10$.

Thus the orbits may be classified as follows:

- $L=0$ : The saddle points $(R, \Theta)=(0,0),(2,0),(2, \pi / 2)$, and the orbit $R=2$, $0<\Theta<\pi / 2$. (Note that from (4.15), whenever $R=2, \gamma=-1$, so that these saddle points will correspond to the unstable equilibrium points $(\alpha, \beta, \gamma)=$ $(0,0,(C-1) /(C+1)),(0,2,-1),(2,0,-1)$ of the three-dimensional system $(4.1)-(4.3)$.

- $0<L<L_{1}$ : Closed orbits for $R<2$, open orbits for $R>2$.

- $L=L_{1}$ : Center at $R=R_{1}, \Theta=\pi / 4$ and an open orbit in the region $R>2$.

- $L_{1}<L \leq 1 / \sqrt{C}$ : Only open orbits in the region $R>2$.

- $1 / \sqrt{C}<L<L_{2}$ : Only closed orbits in the region $R>2$.

- $L=L_{2}$ : Center at $R=R_{2}, \Theta=\pi / 4$.

- $L>L_{2}$ : No orbits.

From (4.22) we can also see that $\frac{d \Theta}{d u}$ will be zero if $C u^{6}+3 u-2=0$ or $u=u_{1}, u_{2}$. Then the extreme values of $\Theta$ on the orbit are given by

$$
\sin 2 \Theta_{e}=L / L_{i}, \quad i=1,2,
$$

i.e.,

$$
\begin{aligned}
\Theta_{\max } & =\pi / 2-\frac{1}{2} \arcsin \left(L / L_{i}\right), \\
\Theta_{\min } & =\frac{1}{2} \arcsin \left(L / L_{i}\right) .
\end{aligned}
$$

A typical phase diagram, both in three-dimensional phase space and as projected on the $\alpha \beta$-plane, is shown in Figure 9 for $C=10$.

5. Application to the leapfrog method. Having studied the dynamics of the amplitudes of the two interacting waves in general, we can now relate it to the actual numerical results obtained when solving (2.5) and (2.6). It should be remembered that the values of $y^{1}$ and $x^{1}$ must be found by using some one-step method like the Euler method or one of the Runge-Kutta methods before the leapfrog method can start. Because of this, the computational wave is present from the outset, as we will demonstrate below.

Copyright (C) by SIAM. Unauthorized reproduction of this article is prohibited. 

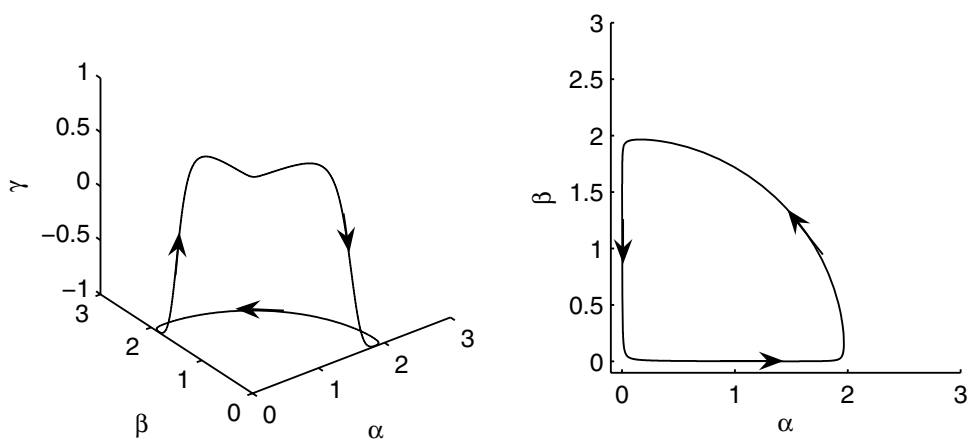

FIG. 10. Orbit in three dimensions, and projected on the $\alpha \beta$-plane, for an Euler starter and $\tau=0.2, \epsilon=0.025, \omega=1, x^{0}=0, y^{0}=0.5$.

To find the initial amplitudes $\alpha(0)=\alpha_{0}$ and $\beta(0)=\beta_{0}$, as well as the initial phase angles $\theta_{0}$ and $\phi_{0}$ (and hence also $\gamma_{0}$ ), we put

$$
x^{n}=\alpha_{0} \cos \left(\frac{\Omega n \tau}{2}+\theta_{0}\right)+(-1)^{n} \beta_{0} \cos \left(\frac{\Omega n \tau}{2}+\phi_{0}\right)
$$

for $n=0,1,2$, and 3. (Here we assume that $\epsilon$ is small enough so that the functions depending on $T_{1}$, i.e., $\alpha, \beta, \theta$, and $\phi$, do not change significantly over three time steps.) Note that $x^{0}$ and $y^{0}$ are the given initial conditions, and $x^{1}, x^{2}$, and $x^{3}$ depend on the actual starter scheme used. By solving for $\alpha_{0} \cos \theta_{0}, \alpha_{0} \sin \theta_{0}, \beta_{0} \cos \phi_{0}$, and $\beta_{0} \sin \phi_{0}$ first, we need only solve a linear algebraic system. Eventually we can then find $R_{0}$, $\Theta_{0}$, and $\gamma_{0}$, and hence the orbit parameters $C$ and $L$.

For $\tau=0.2, \epsilon=0.025, \omega=1, x^{0}=0, y^{0}=0.5$, and an Euler starter, the initial amplitudes and phases were

$$
\begin{aligned}
\alpha_{0} & =0.5089, \\
\beta_{0} & =0.0040, \\
\theta_{0} & =-1.5718 \text { radians, } \\
\phi_{0} & =-1.4428 \text { radians. }
\end{aligned}
$$

Thus the computational wave has a small but significant amplitude $\beta_{0}$ initially. The initial orbit polar coordinates are then

$$
\begin{aligned}
R_{0} & =0.5089, \\
\Theta_{0} & =0.0079 \text { radians, } \\
\gamma_{0} & =0.9669,
\end{aligned}
$$

from which the orbital parameters of this numerical solution can be calculated as $C=88.8002$ and $L=0.00011453$. The resulting orbit and its projection in the $\alpha \beta$-plane are shown in Figure 10.

We also used both a second order and a fourth order Runge-Kutta starter. The initial phases and amplitudes for these are given in Table 1.

The corresponding orbits are shown in Figures 11 and 12.

When comparing Figures 10, 11, and 12, we note that the projection of the orbit on the $\alpha \beta$-plane looks virtually the same in all three cases, but that the full 
TABLE 1

Orbital parameters for different starter schemes.

\begin{tabular}{|l|c|c|c|c|c|c|c|c|c|}
\hline Starter & $\alpha_{0}$ & $\beta_{0}$ & $\theta_{0}$ & $\phi_{0}$ & $R_{0}$ & $\Theta_{0}$ & $\gamma_{0}$ & $C$ & $L$ \\
\hline 2nd order RK & 0.5038 & 0.00117 & -1.5715 & 1.2499 & 0.5038 & 0.0023 & 0.8019 & 13.4768 & 0.000083 \\
\hline 4th order RK & 0.5038 & 0.00132 & -1.5722 & 1.0015 & 0.5038 & 0.0026 & 0.4215 & 3.6407 & 0.000160 \\
\hline
\end{tabular}
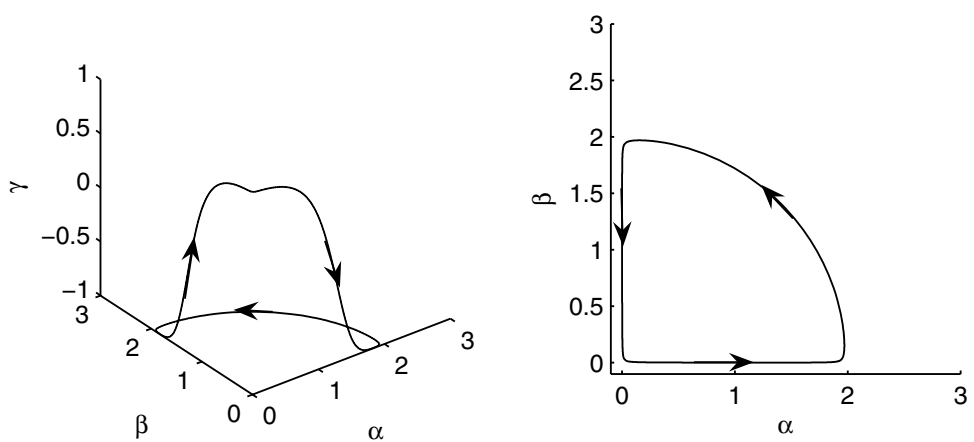

FIG. 11. Orbit in three dimensions, and projected on the $\alpha \beta$-plane, for a second order RungeKutta starter and $\tau=0.2, \epsilon=0.025, \omega=1, x^{0}=0, y^{0}=0.5$.
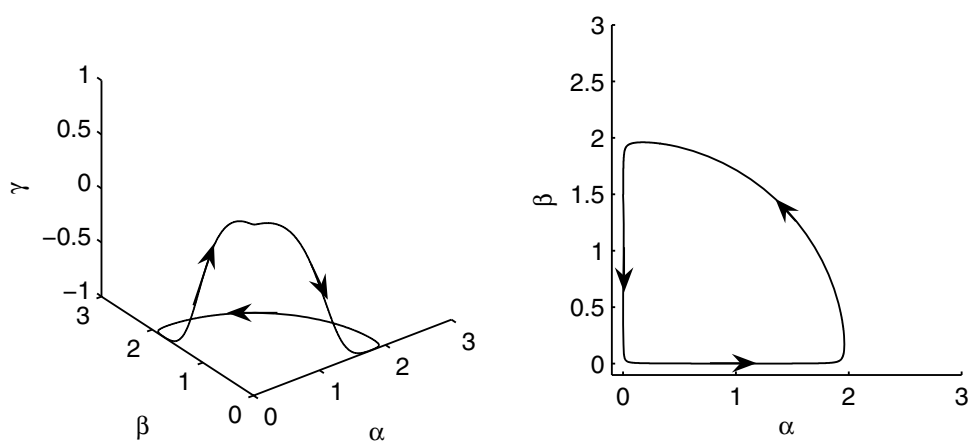

FIG. 12. Orbit in three dimensions, and projected on the $\alpha \beta$-plane, for a fourth order RungeKutta starter and $\tau=0.2, \epsilon=0.025, \omega=1, x^{0}=0, y^{0}=0.5$.

three-dimensional orbit varies significantly. The more inaccurate the starter scheme is, the more variation there seems to be in $\gamma$, and hence in the phase angles $\theta$ and $\phi$.

From the actual orbits it is easy to find the point where the computational wave starts to grow. Cai et al. [1] calculated that this happens when $\alpha=\sqrt{0.8}=0.8944$. Our nonlinear analysis allows us to be more precise by finding the lowest point of a particular orbit. In fact, we can already see that the computational wave is initially damped, until the damping eventually becomes an amplification. In Table 2 we list, for each of the three starter schemes and the parameter values and initial conditions as before, the point $\alpha=\alpha_{1}^{*}$, where the amplitude $\beta$ of the computational wave reaches a minimum value of $\beta^{*}$. At this point the computational wave starts growing, albeit very slowly at first. We also list the point $\alpha=\alpha_{2}^{*}$ where the amplitude $\beta$ first exceeds the value of 0.01 .

Copyright (C) by SIAM. Unauthorized reproduction of this article is prohibited. 
TABLE 2

The point $\alpha=\alpha_{1}^{*}$ on the orbit where $\beta$ reaches its minimum $\beta^{*}$, together with the value $\alpha_{2}^{*}$ of $\alpha$, where $\beta$ first exceeds 0.01 for the three different starter schemes, and $\tau=0.2, \epsilon=0.025, \omega=1$, $x^{0}=0, y^{0}=0.5$.

\begin{tabular}{|l|c|c|c|}
\hline Starter & $\alpha_{1}^{*}$ & $\alpha_{2}^{*}$ & $\beta^{*}$ \\
\hline Euler & 1.4450 & 1.8866 & 0.000996 \\
\hline 2nd order RK & 1.2507 & 1.9043 & 0.000465 \\
\hline 4th order RK & 1.1065 & 1.8655 & 0.000713 \\
\hline
\end{tabular}

We see that $\alpha_{1}^{*}$ is sensitive for the particular starter scheme used, whereas $\alpha_{2}^{*}$ does not vary very much. From Table 1 we see that $C$ decreases with the accuracy of the initial values $x^{1}$ and $y^{1}$. It is worthwhile to note at this point that if $C=0$ (and therefore $\gamma=-1$ throughout), then if we consider an orbit close to $\beta=0$, by putting $\beta=\epsilon \ll 1$, then (4.2) becomes

$$
\epsilon^{\prime}=r \epsilon\left(5 \alpha^{2} / 4-1\right)
$$

showing that $\alpha_{1}^{*}$ would take on the value of $\sqrt{0.8}$ predicted by Cai et al. Their value is therefore a limiting value. In practice, growth of the computational wave starts a bit later. In practical terms, for the type of accuracy expected from a second order method and a time step of 0.2 , the computational wave will start to cause trouble only when its amplitude becomes larger than about 0.01 , i.e., about $0.5 \%$ of the limit circle amplitude. This does not happen before the solution gets very close to the limit cycle, i.e., at about $\alpha=1.9$. Up to then the effect of $\beta$ in (3.22) and (3.24) is negligible, so that the phase angle $\theta$ is virtually constant, and the amplitude of the solution is given by (4.6), which is as near to the analytic solution as can be expected from a second order method. In other words, the numerical solution is quite sound and useful until it very nearly reaches the limit cycle.

We may also note from the phase diagram in Figure 9 that when we start with initial conditions which leads to an initial amplitude larger than that of the limit cycle, the open orbits in the region $R>2$ imply eventual blowup. To test this, we used the parameter values we used before, but changed the initial values to $x^{0}=5$, $y^{0}=0$. With a second order Runge-Kutta starter, the numerical results are shown in Figure 13.

The computational wave is clearly seen to become significantly large quite early, causing a blowup of the solution at about 50 time units. The orbit parameters for these parameter values and initial conditions turn out to be $C=3.1070 \times 10^{-6}$ and $L=5.2642$. For this low value of $C$, closed orbits in the region $R>2$ start only at about $L=567$, so that this orbit is definitely open. Figure 14 shows this orbit, both in three dimensions and projected on the $\alpha \beta$-plane. The point on this orbit where $\beta$ is a minimum is at $\alpha=\alpha_{1}^{*}=6.82$. Since the initial point on this orbit is further along, the computational wave is amplified from the very start, where it already has an amplitude of 0.08. Thus, for these particular initial conditions, the numerical solution is useless.

Finally (4.12) implies that there is another set of planar orbits besides $\gamma=-1$, namely, those on the plane $\gamma=1(C=\infty)$. Then, according to (4.11), $\Theta^{\prime}<0$ on $(0, \pi / 2)$, and hence $\beta$, monotonically decreases for all orbits on this plane. Thus the computational wave will cause no problems as long as $\gamma=1$ (i.e., $\theta=\phi$ ). Unfortunately these orbits are structurally unstable. The smallest deviation from $\gamma=1$ will move the solution to one of the other $C$-surfaces, and hence lead to growth of $\beta$.

Copyright $\odot$ by SIAM. Unauthorized reproduction of this article is prohibited. 


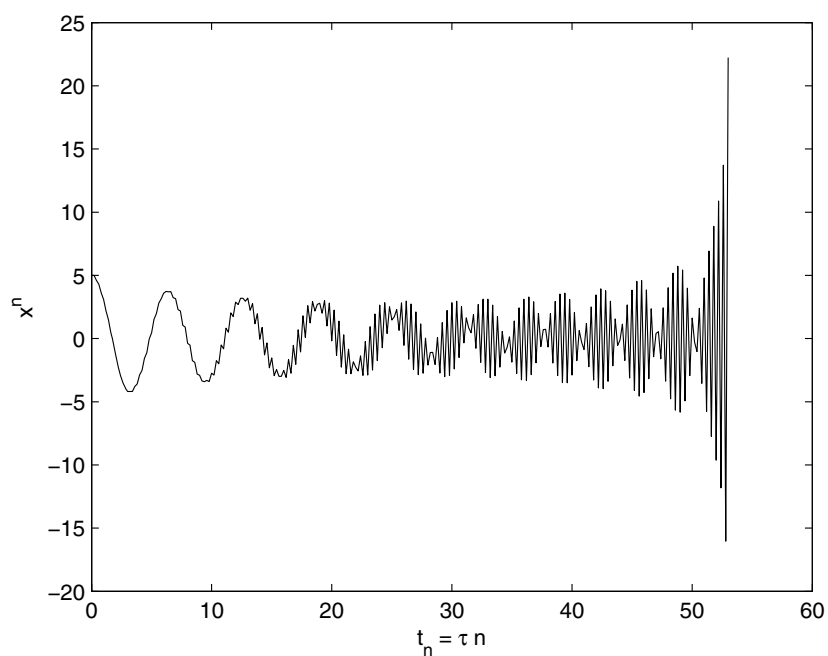

FIG. 13. Results when running the difference system (2.5), (2.6) with $\epsilon=0.025, \tau=0.2$, and $x^{0}=5, y^{0}=0$.
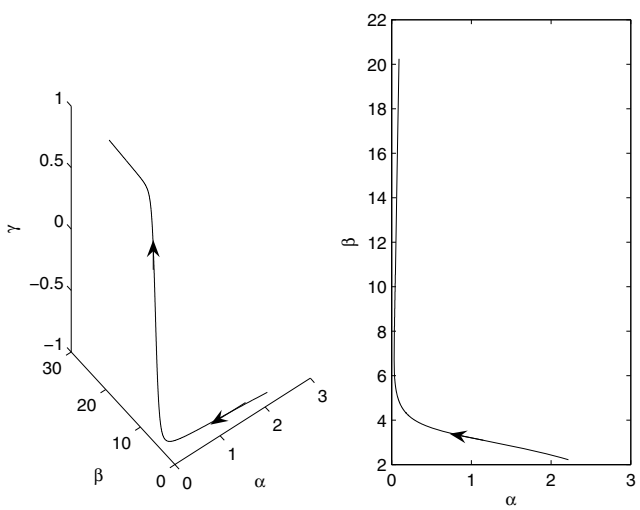

FIG. 14. Orbit in three dimensions, and projected on the $\alpha \beta$-plane, for a second order RungeKutta starter and $\tau=0.2, \epsilon=0.025, \omega=1, x^{0}=5, y^{0}=0$.

6. Discussion. In this paper we demonstrated that attempts to calculate the solution of the van der Pol equation by means of several Nyström methods of different orders lead to nonlinear instabilities caused by sudden growth of a high frequency mode. This high frequency induced (HFI) instability can be delayed simply by decreasing the time step. Thus it is always possible to calculate the limit cycle accurately, provided a small enough time step is used. Time step restrictions get more severe as the order of the method is increased.

As far as the analysis is concerned, we first improved upon the work done by Cai et al. [1], in the sense that by doing a full nonlinear analysis of the amplitude dynamics of the second order Nyström difference scheme (2.5), we were able to fully explain the observed spurious amplitude modulation imposed by it, and also to assess the extent to which this scheme could still be useful as a second order numerical method.

Copyright (C) by SIAM. Unauthorized reproduction of this article is prohibited. 
We also demonstrated the use of a discrete multiple scales technique combined with a phase plane analysis to analyze such a nonlinear difference scheme. These techniques will certainly also be applicable to other numerical methods for the van der Pol and other nonlinear differential equations, and indeed to nonlinear models which are discrete to start with.

The fact that leapfrog time discretizations could cause spurious behavior has been known for some time [7, 8, 11], and Sanz-Serna [7] and Sanz-Serna and Vadillo [8] did a phase plane analysis in some simpler cases. Of course, in the case of (2.5) the problem is much more complicated, since it is not the behavior of the solution itself which needs to be analyzed (as in the cases studied by Sanz-Serna and his coworkers), but rather that of its amplitude, which makes it necessary to use a discrete multiple scales method first.

In a case such as Duffing's equation, which models oscillations but does not have a limit cycle, we found that the HFI instabilities were less severe, in the sense that it seems to be totally absent in the case of the second order Nyström scheme, although still present in the case of the higher order methods.

We also observed HFI instability where Nyström methods were applied to other cases where limit cycles occurred in the dynamics of the solutions. One such case was a predator-prey system described in [5, Chapter 3, section 3.3]. An analysis of these and other cases is currently under way and will be discussed in forthcoming papers.

\section{REFERENCES}

[1] D. Cai, A. Aoyagi, And K. ABe, Parametric excitation of computational mode of the Leapfrog scheme applied to the van der Pol equation, J. Comput. Phys., 107 (1993), pp. 146-151.

[2] E. Hairer, S. P. Nørsett, And G. Wanner, Solving Ordinary Differential Equations I, Springer-Verlag, Berlin, 1988.

[3] D. W. Jordan And P. Smith, Nonlinear Ordinary Differential Equations, 3rd ed., Oxford University Press, Oxford, UK, 1999.

[4] G. M. Moremedi And D. P. Mason, Analysis of the limit cycle of a generalized van der Pol equation by a time transformation method, Quaest. Math., 17 (1994), pp. 349-380.

[5] J. D. Murray, Mathematical Biology. I, 3rd ed., Springer-Verlag, New York, 2002.

[6] A. H. NAYfeh, Perturbation Methods, Wiley, New York, 1973.

[7] J. M. SANZ-SERnA, Studies in numerical nonlinear instability I. Why do leapfrog schemes go unstable?, SIAM J. Sci. Comput., 6 (1985), pp. 923-938.

[8] J. M. SAnz-SERna AND F. VAdillo, Studies in numerical nonlinear instability III. Augmented Hamiltonian systems, SIAM J. Appl. Math., 47 (1987), pp. 92-108.

[9] S. W. Schoombie, A Discrete Multiple Scales Analysis of a Discrete Version of the Kortewegde Vries Equation, Report NA/109, University of Dundee, 1987.

[10] S. W. Schoоmbie, A discrete multiple scales analysis of a discrete version of the Kortewegde Vries equation, J. Comput. Phys., 101 (1992), pp. 55-70.

[11] D. M. Sloan And A. R. Mitchell, On nonlinear instabilities in leap-frog finite difference schemes, J. Comput. Phys., 67 (1986), pp. 372-395.

[12] B. VAN DER POL, On a type of oscillation hysteresis in a simple triode generator, Philos. Mag., 43 (1922), pp. 177-193.

Copyright (C) by SIAM. Unauthorized reproduction of this article is prohibited. 